\title{
Publisher Correction: Migrasome formation is mediated by assembly of micron-scale tetraspanin macrodomains
}

Yuwei Huang, Ben Zucker, Shaojin Zhang, Sharon Elias, Yun Zhu, Hui Chen, Tianlun Ding, Ying Li, Yujie Sun, Jizhong Lou, Michael M. Kozlov (D) and Li Yu (i)

Correction to: Nature Cell Biology https://doi.org/10.1038/s41556-019-0367-5, published online 1 August 2019

In the version of this article originally published, there were several errors in the author information. The email for co-corresponding author Michael M. Kozlov was missing from the 'author information' section in the HTML version of the paper. The corresponding author sentence in the HTML should read "Correspondence to Michael M. Kozlov or Li Yu." Furthermore, an equal contribution statement was missing for co-authors Yuwei Huang, Ben Zucker and Shaojin Zhang. This should be affiliation footnote number 6 and read "These authors contributed equally: Yuwei Huang, Ben Zucker, Shaojin Zhang." The errors have been corrected in the HTML and PDF versions of the paper.

Published online: 21 August 2019

https://doi.org/10.1038/s41556-019-0389-z

\section{Publisher Correction: HER2 joins AKT to inhibit STING immunity}

Ian D. Odell and Richard A. Flavell D

Correction to: Nature Cell Biology https://doi.org/10.1038/s41556-019-0368-4, published online 1 August 2019

In the version of this paper originally published, Fig. 1 was incorrectly titled "ERB2 and HER2 in immunity." The Fig. 1 heading should be "Cellular trafficking of EGFR and HER2 during viral infection." The error has been corrected in the HTML and PDF versions of the paper.

Published online: 23 August 2019

https://doi.org/10.1038/s41556-019-0395-1 